\title{
Analysis on the Motion Characteristics of the Crank-group Driving Mechanism by Graphical Method
}

\author{
Wang Yuqi \\ College Of Mechanical Engineering, \\ Xijing University, \\ Xi'an, China, \\ E-mail:wangyuqi1117@126.com
}

\author{
Sheng Shangxiong \\ College Of Mechanical Engineering, \\ Xijing University, \\ Xi’an, China, \\ E-mail:972118362@qq.com
}

\begin{abstract}
The new transmission mechanism crank-group driving mechanism is mainly used in some special light industrial machinery. Paper drew the mechanism's trajectory curve of the structure unit by a new way-the graphical method, analyzed it related to the trajectory function, and then studied the motion characteristics of the whole mechanism. The study shows that all the cranks' motion on the crank-group driving mechanism are synchronous and uniform at any time, the connecting rod truss composed of the parallel double crank mechanism is doing translation from beginning to the end. The conclusions are consistent with the previous research; it validated the correctness of the foregoing research, and then laid the theoretical foundation for the mechanisms widely used in future.
\end{abstract}

Keywords-multi-crank mechanism; structural unit; graphical method; trajectory function; characteristic analysis

\section{INTRODUCTION}

The new multi-crank transmission mechanism crank-group driving mechanism is mainly used in some special light industrial machinery (such as some packaging machinery, tobacco machinery, special drilling machine and printing machine, etc.), to obtain same speed between the multiple parallel axis, and transfer the torque which is not too big[1]. Compared with the traditional way of transmission such as gear, toothed belt or chain transmission, the crank-group driving mechanism can greatly shorten the transmission chain, and it has many characteristics, such as the structure is simple and the price is low. At present, the domestic and foreign research on the multi-crank mechanism is very rare, in the research literature, a foreign mechanical device which is called trisector and the domestic design about the multi-crank shaft drilling machine is directly related to the mechanism[2-3]. Domestic research involves the mechanism mainly focused on the feasibility and analysis of the mechanism, its basic configuration, basic theory and characteristic, its design method, etc.[4-6].

In actual production, when we study the movement of the mechanism, we usually not only need to know the movement of the mechanism in a certain position, but also often demand to know the motion of the mechanism in the whole motion cycle. Through drawing mechanism's motion diagram by

This work was financially supported by the Science Foundation of Xijing University (XJ160124). graphical method, we can get the mechanism's any instantaneous motion parameters, and can observe the movement situation of the mechanism visually and clearly. Then we can have a comprehensive understanding of the movement characteristic of the mechanism[7]. This paper will analyze and study the movement characteristics of the crankgroup driving mechanism by graphical method.

We analyze and observe the changes of displacement of crank-group driving mechanism throughout the course of motion by the graphical method. Suppose that the known crank rotates counterclockwise with equal angular velocity $\omega(n=600 n / \mathrm{min}$ that is $\omega=20 \pi \mathrm{rad} / \mathrm{s})$. First, you can make a motion chart of a structural unit (As shown in Fig.1, it is a structural unit of crank-group driving mechanism in the coordinate system, crank 1 is the driving crank, and crank 3 is a driven crank) of the mechanism.

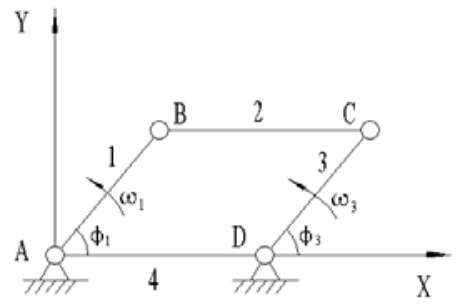

Fig. 1. The structural unit in the coordinate system

Speaking of the structure of the crank-group driving mechanism, it is composed of a plurality of parallel doublecrank mechanism, and it is obtained by combination and stacking in various ways. The motions of these repetitive structures are exactly the same, so we can know the characteristics of motion of the whole mechanism through the study on one of the structural units. There're displacement components for displacement of the driven crank on the $\mathrm{X}$ axis and $\mathrm{Y}$ axis, so its displacement can be divided into two components separately on the $\mathrm{X}$ axis and $\mathrm{Y}$ axis for the purpose of research. 


\section{THE DISPLACEMENT COMPONENT $S_{C Y}$ ON THE $Y$ AXIS}

As shown in Fig. 2, we divide the trajectory of the driving crank 1 into 16 portions along the circumference, and find out a series of locations of point $C$ on the driven crank 3 which has the one-to-one correspondence with them by graphical method. Then the distances such as $C_{1} C_{2}, C_{1} C_{3}, C_{1} C_{4}$ and so on are exactly the displacements of the corresponding point $C$ on the driven crank 3 from its starting position $C_{1}$ when the crank 1 in various locations (that means within each certain time). We can get the displacement component of it along the $\mathrm{Y}$ axis, and the displacement scale is equal to the scale of mechanism chart $\mu_{s}=\mu_{L} \quad$ In Fig.2, the intercepted line segment on the horizontal axis with length of $L(L=96 \mathrm{~mm})$ represents the rotating time of a whole circle for the crank, and its time scale is $\mu_{t}$; the crank rotates with equal angular velocity, so the abscissa also represents the rotation angle $\varphi$ of crank 3 , then the scale is $\mu_{\varphi} \cdot T=\frac{t}{n}$, supposing that then $T=\frac{t}{n}=\frac{60}{600}=0.1 s$.

In the formula above, $n$ represents the rotation number of crank per minute;

$$
\begin{aligned}
& \mu_{t}=\frac{T}{L}=\frac{0.1}{96}=\frac{1}{960}(\mathrm{~s} / \mathrm{mm}) \\
& \mu_{\varphi}=\frac{2 \pi}{L}=\frac{2 \pi}{96}=\frac{\pi}{48}(\mathrm{rad} / \mathrm{mm})
\end{aligned}
$$

In Fig.2, the positive and negative of displacement $S_{C Y}$ can represent the position of the point C in the mechanism: when the displacement $S_{C Y}$ is positive, it means that the position of the point $\mathrm{C}$ is above the $\mathrm{X}$ axis; when the displacement $S_{C Y}$ is negative, it means that the position of the point $\mathrm{C}$ is below the $\mathrm{X}$ axis; when the displacement $S_{C Y}$ is zero, it means that the position of the point $\mathrm{C}$ is just on the $\mathrm{X}$ axis, crank and the connecting rod are collinear at this time.

\section{ANALYSIS ON THE LAW OF THE DISPLACEMENT COMPONENT $S_{C Y}$ ON THE $Y$ AXIS}

The change of displacement for driven crank 3 on the $Y$ axis in the movement can be seen from Fig. 2, and it will undergo cyclical changes along with the movement. We only drew out the movement of a cycle in the picture. The displacement change curve of driven crank 3 along the axis direction is very similar to the image of sinusoidal functions which we are familiar with. The amplitude of the displacement function $\mathrm{A}$ along the $\mathrm{Y}$ axis direction is equal to the stroke of crank in one quadrant along the $\mathrm{Y}$ axis, which is equal to the length of crank. The displacement function is an odd function, cycle of the function is $2 k \pi$, and the least positive cycle is $2 \pi, k$ is a positive integer among them[8]. The displacement function along $\mathrm{Y}$ axis can be expressed as $S_{C Y}=A \sin \varphi=l_{1} \sin \varphi$, (amplitude of the function $A=l_{1}$, $\varphi=\phi_{3}=\phi_{1}$ among them). Describing a series of position of driven crank 3 by drawing, we can see that the motion trajectory of crank 3 is a circle from the displacement component diagram. Assuming that the coordinates of the point $D$ is $\left(x_{D}, 0\right)$, then the trajectory of point $C$ on the driven crank 3 meets the trajectory equation of the $\operatorname{circle}\left(x-x_{D}\right)^{2}+y_{D}^{2}=l_{1}^{2}$.

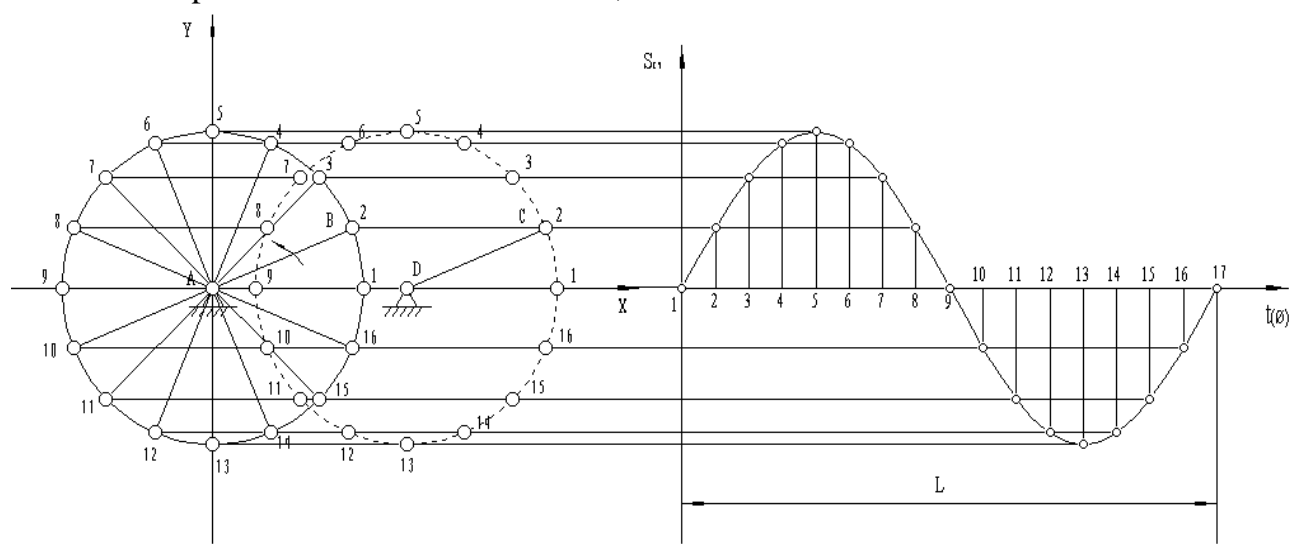

Fig. 2. The displacement component $S_{C Y}$ on the $Y$ axis

At the same time, we can also find this rule: If the displacement component function of the point $\mathrm{C}$ on the crank 3 along the $\mathrm{Y}$ axis in a cycle of movement range is divided into, II , III, IV four intervals, it have relationship of one-to-one correspondence with the I, II, III, IV quadrants (as shown in
Fig. 3)in the coordinate system. Then we will see that the displacement of point $\mathrm{C}$ on the crank 3 is increased gradually in the first and third quadrant, but a positive, a negative (as shown in the shaded area in Fig.3)[8]. 

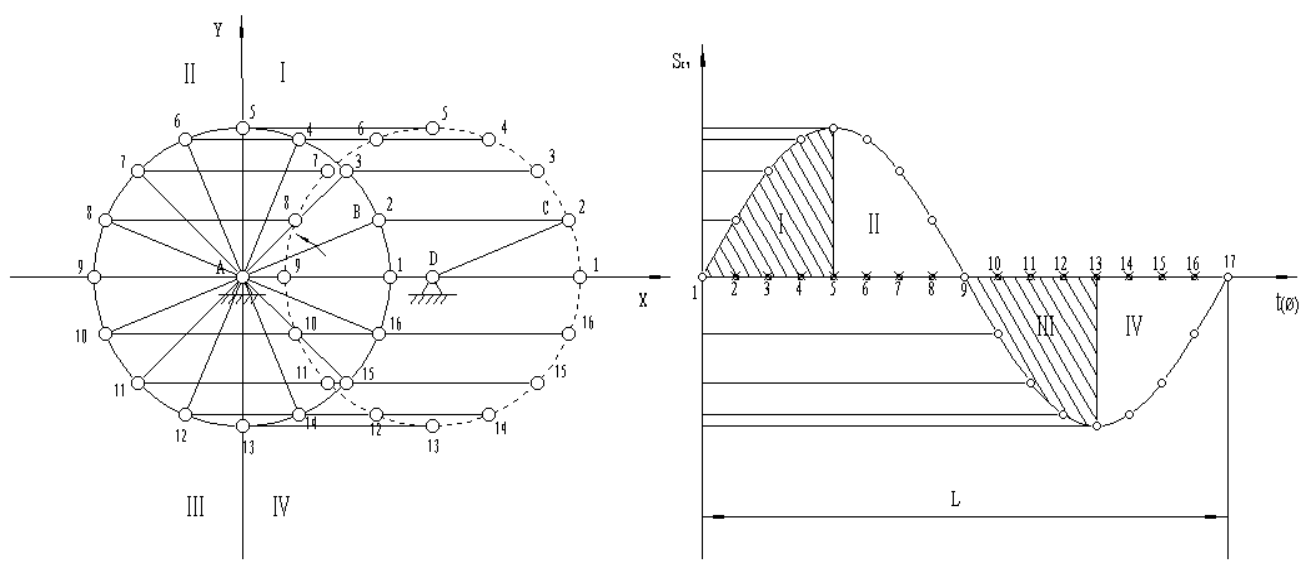

Fig. 3. The change of displacement component $S_{C Y}$ on the $Y$ axis

While in the second and fourth quadrants, the displacement of point $\mathrm{C}$ on the crank 3 is also decreasing and along the positive and negative directions respectively (as shown in the blank area in Fig.3).Meanwhile, we can see that the function has monotonicity in the I, II, III, IV four interval. In the I, IV interval, the function showed a trend of increasing, in the II , III interval, the function showed a trend of decreasing. We can infer the monotone interval of the displacement component function according to the periodic and monotone interval of the sine function.

The analysis shows that the displacement on the axis is similar to the $\mathrm{Y}$ axis.

\section{THE MOVEMENT CHARACTERISTIC CURVE OF THE DRIVEN CRANK}

Overlay the displacement component of the crank 3 along the $\mathrm{X}$ axis and the $\mathrm{Y}$ axis, we can get the function that $S_{C Y}=A \sin \varphi=l_{1} \sin \varphi$ which is similar to the sine function and the function that $S_{C X}=A \cos \varphi=l_{1} \cos \varphi$ which is similar to the cosine function. The superimposed effect of them is a circle, and the radius of the circle is $l_{1}$, the center of the circle is $\left(x_{D}, 0\right)$. Therefore, the trajectory equation of driven crank 3 is $\left(x-x_{D}\right)^{2}+y_{D}^{2}=l_{1}^{2}$, which is consistent with the preceding observations.

The speed and acceleration curve shows that what the mechanism does is uniform motion, so they are straight lines which are parallel to the $\mathrm{Y}$ axis ( $C$ is a positive constant) $v=c, a=0$. Both the velocity and acceleration function are constant function, they do not change with time.

\section{CONCLUSION}

Draw the trajectory curve of the structural unit of the crankgroup driving mechanism by graphical method, and express it by mathematical function to analyze and study. Through the kinematics characteristic of the structural unit, we can get the kinematics characteristic of the crank-group driving mechanism.

What needs to explain is that, all the cranks' motion on the crank-group driving mechanism are synchronous and uniform at any time, the connecting rod truss composed of the parallel double crank mechanism is doing translation from beginning to the end.

The conclusion of this paper is consistent with the research conclusion in reference [1], and it validates the correctness of the foregoing research achievements. It can also provide certain theoretical basis for deep study on the mechanism in the future.

\section{REFERENCES}

[1] Wang Yuqi, Cao jujiang. Study on the motion feature of crank-group driving mechanism [J]. Journal of mechanical transmission, 2013, 37(10):79-81.

[2] Lyndon O. Barton. Mechanism analysis of a trisector [J].Mechanism and Machine Theory, 2008, 43(2).

[3] Yang Wen. Design of axial drilling machine with multicranks [J]. Science and technology information development and economic, 2001, 11(2):75-76.

[4] Ju-jiang, Cao; Yu-qi, Wang. Feasibility research of crank-group driving mechanism. Source: Applied Mechanics and Materials, v215-216, p959963, 2012, Advances in Design Technology.

[5] Ju-jiang, Cao; Yu-qi, Wang. Study on the basic configurations of crankgroup driving mechanism. Source: Applied Mechanics and Materials, v246-247, p141-144, 2013, Computer-Aided Design, Manufacturing, Modeling and Simulation II.

[6] Ju-jiang, Cao; Yu-qi, Wang; Ji-wei, Sun. Basic research of crank-group driving mechanism Source: Advanced Materials Research, v538-541, p701-704, 2012, Materials Processing Technology II.

[7] Sun heng, Chen zuomo, Ge wenjie, etc. Mechanical principles (7th edition)[M]. Beijing: Higher Education Press, 2006.

[8] Tongji University. Higher mathematics (6th Edition) [M].Beijing: Higher Education Press, 2007. 